\title{
SIMULAÇÃO FLUIDODINÂMICA DE UM REATOR CONTÍNUO DE PRODUÇÃO DE CELULOSE
}

\author{
Diego do Mato Lara1, Ivo Neitzel 2*, Jéssica da Luz Maeiski3 \\ 1 - Faculdade de Telêmaco Borba - FATEB - PR \\ 2 - Faculdade de Telêmaco Borba - FATEB - PR, ivo.neitzel@fatebtb.edu.br \\ 3 - Faculdade de Telêmaco Borba - FATEB - PR
}

\begin{abstract}
Durante o processo de polpação kraft, as fibras de celulose são liberadas de uma matriz de lignina de um cavaco, o referido processo acontece a elevada temperatura e pressão com a utilização de uma solução alcalina. Uma remoção deficiente de lignina induz a dificuldades operacionais e a queda da qualidade do produto. Pesquisadores especulam que esse problema é devido à distribuição irregular da solução alcalina através do leito de cavacos durante a reação. Uma das questões cientificas que ainda estão abertas é como os fluidos, e a coluna de cavacos se comportam dentro de um reator de cozimento. O objetivo deste trabalho é, implementar um modelo no software Comsol Multiphysics, simulando a dinâmica na qual o fluido escoa e dispersa através do leito, com o intuito de caracterizar os padrões de fluxo os líquidos dentro do reator e também o perfil de pressão do leito de cavacos.

Palavras-chave: simulação, fluidodinâmica, digestor contínuo.
\end{abstract}

\section{INTRODUÇÃO}

O processo Kraft tem sido amplamente estudado nos últimos anos. A maior parte da polpa kraft produzida no mundo atualmente é em digestores [6].

O Brasil possui destaque no setor de produção de celulose e crescentes avanços nas tecnologias justificam a realização deste estudo, o país tem um grande potencial de crescimento ligado a vantagens climáticas, extensão territorial e tecnologia florestal evoluída.

O conhecimento dos aspectos inerentes aos processos internos ao digestor é de grande importância para que os desenvolvimentos na área continuem avançando.

O presente trabalho tem como objetivo caracterizar a fluidodinâmica em um meio poroso compressível. Este estudo é baseado no processo de digestão de cavacos de madeira que ocorrem em um digestor contínuo. A solução chamada tipicamente de licor branco, é uma solução aquosa de soda e sulfeto de sódio em contato com os cavacos a aproximadamente $150^{\circ} \mathrm{C}$ e $700 \mathrm{kpa}$, por aproximadamente 4 horas. Para garantir uma boa transferência de calor e massa para os cavacos durante a reação, os licores do processo são recirculados através do digestor depois de serem aquecidos em um trocador de calor externo [1].

O fluxo de licor internamente ao digestor deveria ser mantido uniforme para que os reagentes possam ser supridos aos cavacos e os subprodutos sejam removidos. Infelizmente esse não é o caso na maioria dos digestores, como indicação de não uniformidade, ocorre a corrosão acelerada, [1], flutuações no número Kappa de saída, que é uma medida da extensão da reação [1], e gradientes de temperatura [1] são evidentes.

Variações espaciais no fluxo de licor resultam em amolecimento dos cavacos conforme a reação progride. $\mathrm{O}$ amolecimento dos cavacos resulta em compactações locais do leito, e a subsequente diminuição da porosidade limita a penetração do licor branco. Esse fenômeno é aumentado a medida que o amolecimento dos cavacos pode causar mudanças no tamanho da distribuição das partículas; isso pode causar uma redução local na porosidade do leito [1]. O entendimento dos fluxos de licor dentro do leito poroso, e a contribuição com o conhecimento relativo aos processos de cozimento no digestor, são as metas deste trabalho.

\section{METODOLOGIA}

A simulação é feita em modo estacionário utilizando:

- Fluidodinâmica em meios porosos - Equações de Brinkman; 
- Módulo matemático - EDP na forma de coeficientes.

A fluidodinâmica é composta por:

- Entrada de licor e cavacos no topo do digestor;

- Tubo interno central para adição de licor de recirculação no interior do digestor;

- Peneiras para extração do licor de recirculação;

- Lavagem contracorrente no fundo do digestor;

- Saída para descarga da polpa e do licor no fundo do digestor.

O processo de cozimento ocorre à temperatura de aproximadamente $150{ }^{\circ} \mathrm{C}$ e pressão de 700 kPa por cerca de quatro horas, com adição de Sulfeto de Sódio e Hidróxido de Sódio, que dissolvem a lignina, a substância que une as células da celulose, e liberam as fibras de celulose. $\mathrm{Na}$ saída do digestor os cavacos são convertidos em uma pasta marrom, denominada de celulose não branqueada. O digestor simulado é do tipo, contínuo, de fase vapor.

A simulação, na sua essência, envolve o (1) - o escoamento fluidodinâmico da solução de reagentes (licor) permeando através de um leito poroso com porosidade e permeabilidade fixas em regime estacionário sem reação química.

O escoamento é classificado como "não Darcy" mas com velocidades baixas, descrito pela equação de Ergun modificada. A pressão exercida sobre os cavacos provoca a deformação do leito e depende de (1) - peso próprio dos cavacos, (2) - empuxo do licor reagente, (3) - atrito junto a parede do digestor, e, (4) - arraste pelo licor (ascendente e descendente).

A descrição clássica para o escoamento de líquidos em meios porosos neste contexto utiliza a Lei de Darcy [2] juntamente com a equação da conservação de massa, válidas para escoamento monofásico e laminar:

$$
\begin{gathered}
\varepsilon_{L} \cdot \frac{\partial \rho}{\partial t}=-\left(\nabla \cdot \rho \cdot \mathbf{v}_{\mathbf{0}}\right) \\
\mathbf{v}_{\mathbf{0}}=-\frac{k}{\mu} \cdot \nabla \wp
\end{gathered}
$$

Este modelo foi modificado por Brinkman para considerar a distorção provocada por efeitos viscosos no perfil de velocidade [2] pela proximidade das paredes por intermédio do termo $\nabla^{2} \mathbf{v}_{\mathbf{0}}$ [4] [8]:

$$
\mathbf{v}_{\mathbf{0}}=-\frac{k}{\mu} \cdot \nabla \wp+k \cdot \frac{\mu_{e f f}}{\mu} \cdot \nabla^{2} \mathbf{v}_{\mathbf{0}}, \text { ou, } \quad \nabla \wp=-\frac{\mu}{k} \cdot \mathbf{v}_{\mathbf{0}}+\mu_{\text {eff }} \cdot \nabla^{2} \mathbf{v}_{\mathbf{0}}
$$

Nesta equação $\mu_{\text {eff }}$ é uma viscosidade efetiva que pode ser diferente de ${ }^{\mu}$ [5]. Para diâmetros de poros muito pequenos a variação de velocidade através dos poros pode ser desprezada [8], e, neste contexto, esta parcela não é considerada. 
A geometria é ilustrada na figura 1. É uma geometria 2D axissimétrica.

Fig 1 - Geometria do digestor.

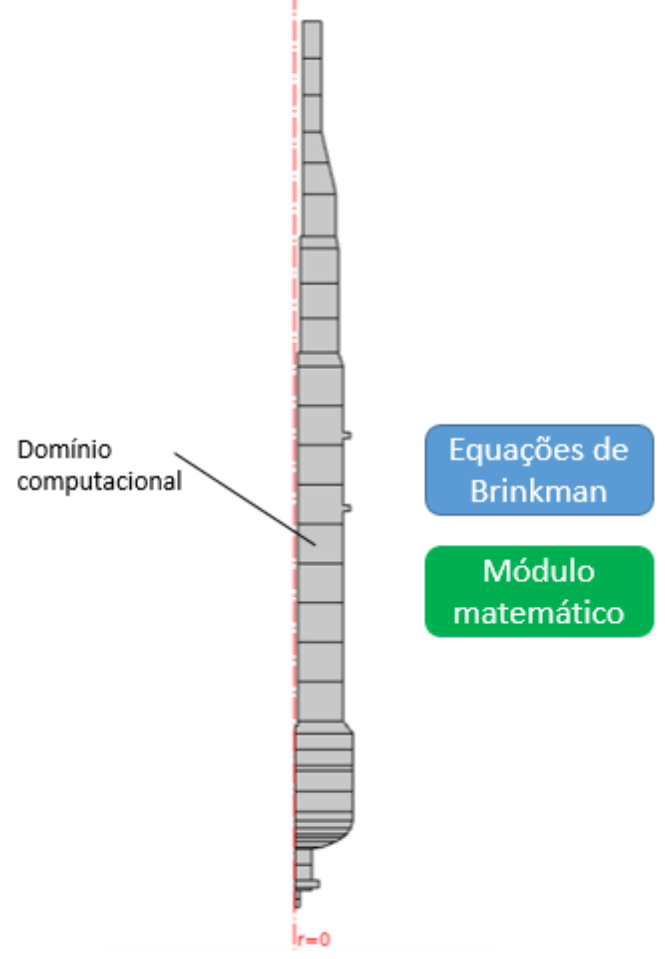

\section{Resultados e discussão}

Os perfis de velocidades mostram o fluxo de licor nas diferentes zonas do digestor. O leito de cavacos foi considerado fixo. No digestor real a velocidade diminui drasticamente no início e depois permanece constante no restante do digestor. As variações verificadas são em parte devido à alteração da área de seção. No início, o digestor é cônico e depois cilíndrico aumentando gradualmente o seu diâmetro.

A figura 2 mostra as linhas de fluxo no interior do digestor. Na base ocorre uma pré-lavagem da polpa em sistema contracorrente. As linhas de fluxo mostram maior escoamento nas entradas e em direção ás zonas de extração de licor.
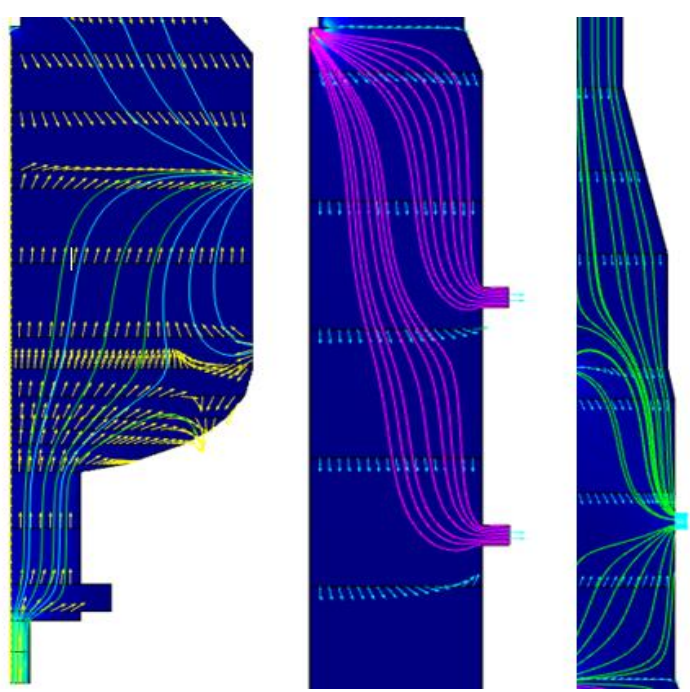

Fig 2 - Linhas de fluxo no digestor da esquerda para direita: fundo, meio e topo.

A velocidade vertical do licor varia à medida que a polpa percorre o perfil do digestor. Esta 
variação de velocidade é causada pela variação do diâmetro do digestor em seu perfil longitudinal, pelas interferências do fluxo de licor adicionado, pelo nível de licor, e pelo peso da coluna. Na figura 3 é possível ver o gradiente de velocidade no digestor.

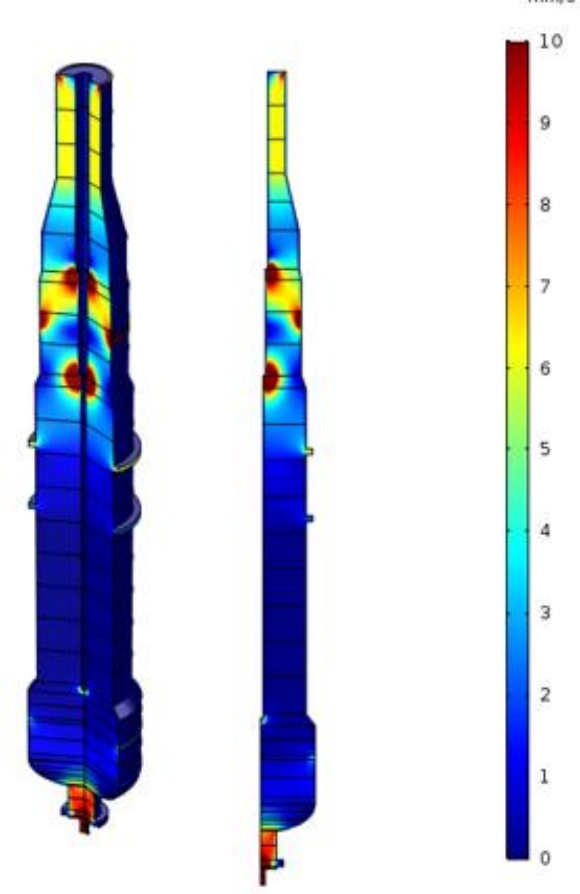

Fig 3 - Perfil de velocidade do fluido ( $\mathrm{mm} / \mathrm{s})$.

O maior gradiente de velocidade pode ser verificado nos pontos de adição e extração dos licores. Próximo das entradas de licor a velocidade é de $10 \mathrm{~mm} / \mathrm{s}$ ou $0,01 \mathrm{~m} / \mathrm{s}$. Abaixo da zona de cozimento a velocidade diminui e se mantém praticamente constante com velocidade de 1 $\mathrm{mm} / \mathrm{s}$ ou $0,001 \mathrm{~m} / \mathrm{s}$.

A figura 4 mostra o perfil de velocidade de escoamento do fluido em sentido vertical no digestor.

Line Graph: Velocity field, z component (mm/s)

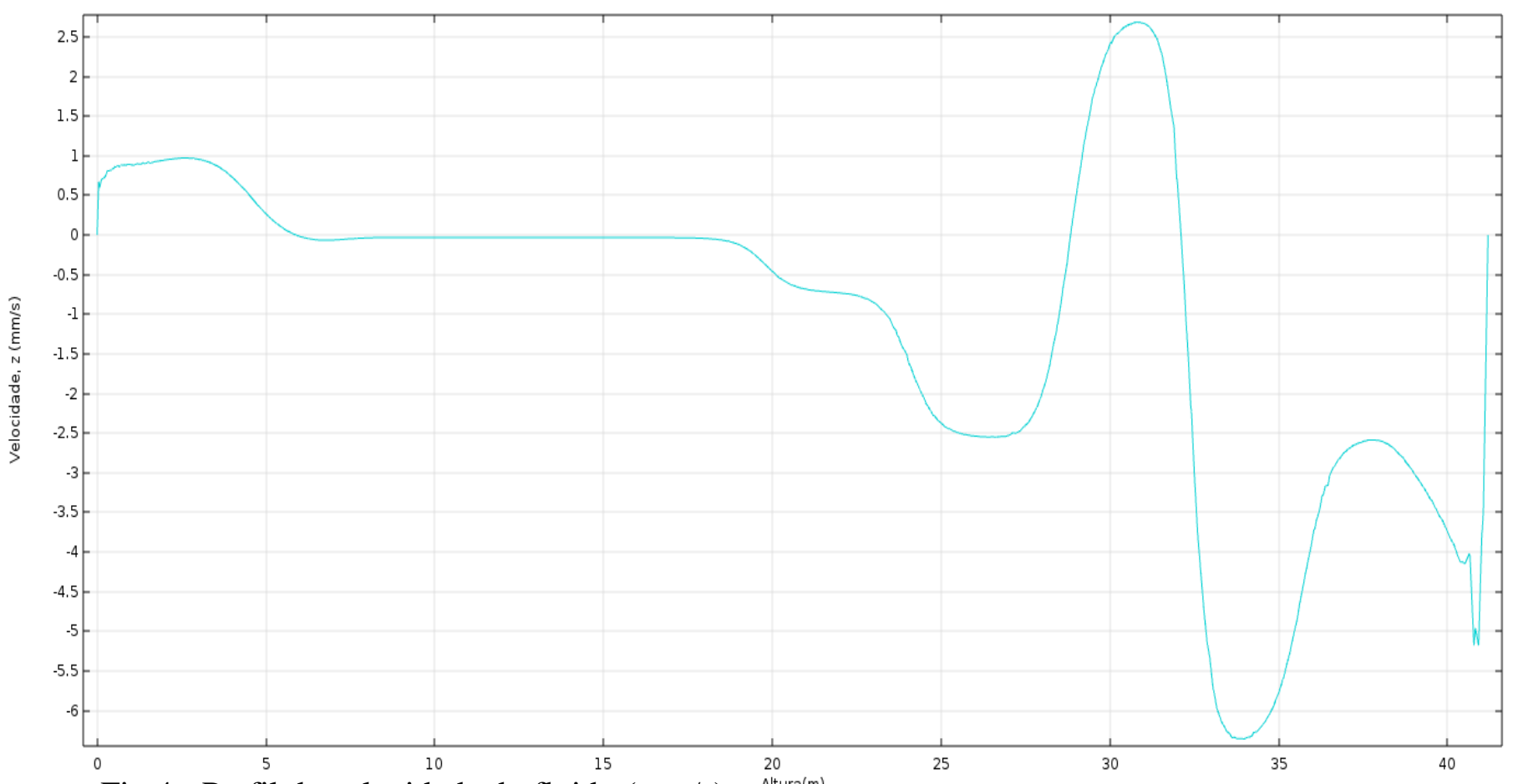

Fig 4 - Perfil de velocidade do fluido $(\mathrm{mm} / \mathrm{s})$. 
No gráfico 4 pode-se notar que a velocidade chega a ultrapassar $6 \mathrm{~mm} / \mathrm{s}$ até na altura de 32,1 metros, o sinal negativo da grandeza indica que o vetor velocidade está no sentido "downflow" (para baixo) no digestor.

A partir da altura de 32,1 metros o vetor muda de sinal, isto indica que o licor que está sendo adicionado na recirculação está fluindo "upflow" no reator, isto pode ser verificado na imagem 4 na representação do topo.

No ponto de aproximadamente $18-7$ metros é a zona de cozimento onde o fluxo de licores está estável fluindo no sentido para baixo com velocidades de $0,032 \mathrm{~mm} / \mathrm{s}$.

A lavagem em contracorrente que ocorre no fundo do digestor é representada no gráfico e o sinal do vetor de velocidade (z) indica o sentido "upflow" do fluido.

A pressão atuante sobre os cavacos foi calculada por meio de um balanço de forças que é a equação de Saltin [7].

Na figura 5 é possível ver o comportamento da pressão no reator, que é maior no centro e vai diminuindo no sentido radial em direção às periferias do digestor.

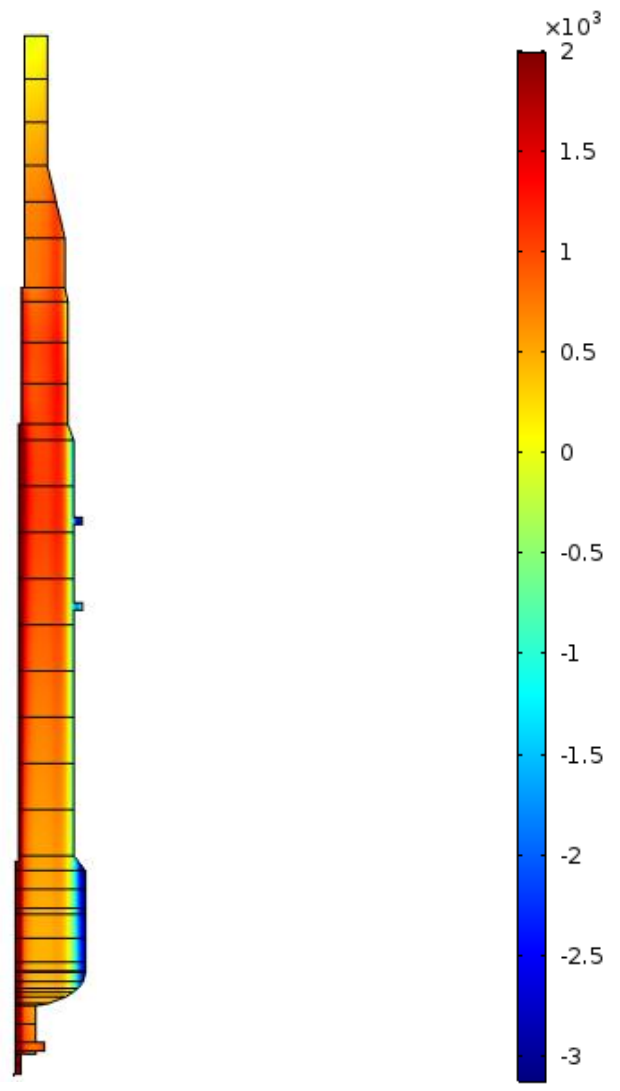

Fig 5 - Perfil pressão dos cavacos $(\mathrm{kPa})$

O gráfico acima corrobora a visão de [3] que há um fluxo preferencial dos cavacos no centro do que nas proximidades da parede do digestor.

\section{CONCLUSÃO}

Foi possível simular a fluidodinâmica utilizando a lei de Darcy modificado por Brinkman, juntamente com o coeficiente de Forchheimer para escoamento em meios porosos.

Pôde-se observar condições do processo real, no que concerne o balanço hidráulico do digestor, que é de suma importância para qualidade do cozimento e boa operação do digestor, bem como a fluidodinâmica dentro de um reator deste tipo. 
Com base nesta simulação obtemos os perfis de escoamento de fluidos, pressão da coluna de cavacos com a equação de Saltin, velocidade dos fluidos e padrões de fluxo.

\section{REFERÊNCIAS}

1. ALAQQAD, Mohammed OM. Characterizing the permeability and dispersion of flows through compressible wood-chip beds. 2011. Tese de Doutorado. University of British Columbia.

2. BSL, R. Byron Bird \& Warren E. Stewart \& Edwin N. Lightfoot; Transport Phenomena, Second Edition, 2002, John Wiley \& Sons, Inc., ISBN 0-471-41077-2.

3. CORREIA, Flávio Marcelo et al. Análise de distúrbios de compactação de cavacos de eucalipto em digestores contínuos fase vapor. 2010.

4. Deen, William M., Analysis of transport phenomena, Oxford University Press, 2nd edition 2012, isbn 978-0-19-974028-4.

5. Delgado, Frank A. Coutelieris \& J.M.P.Q. Delgado; Transport Processes in Porous Media (Series in Advanced Structured Materials), 2012, Springer, ISBN-10= 3642279090, ISBN-13= 978-3642279096.

6. PUOLAKKA, Heidi-Maria; TERVASKANTO, Manne; AHVENLAMPI, Timo. Observation and visualization of the chip compaction disturbances in digester. In: TAPPI Engineering, Pulping \& Environmental Conference. 2005. p. 1-6.

7. SALTIN, J. F. A predictive dynamic model for continuous digesters. In: Tappi Pulping Conference. TAPPI PRESS, 1992. p. 261-261.

8. ZENG, Zhengwen; GRIGG, Reid. A criterion for non-Darcy flow in porous media. Transport in porous media, v. 63, n. 1, p. 57-69, 2006. 